\title{
Distributed Compressive Sensing based Near Infrared and Visible Images Fusion for Face Recognition
}

\author{
Dan Wei \\ Shanghai University of Engineering Science \\ weiweidandana@163.com
}

\begin{abstract}
In this paper, we propose a novel face recognition method based on fusing the near infrared and visible images of face images with distributed compressive sensing. The near infrared image and visible image of one same subject constitute an ensemble. Both images in one ensemble share a common sparse component while each individual image has an innovation component. To better capture the complementary information of the ensemble, the distributed compressive sensing is used to obtain the common component and the innovation component of near infrared and visible image. The obtained common component contains the complementary information of near infrared and visible image effectively. So the sparse coefficients of the common component obtained by distributed compressive sensing can better capture the intrinsic structures of each image and therefore can obtain better performance than that of only using near infrared image or visible image. The experimental results on several benchmark datasets demonstrate the effectiveness of proposed method.
\end{abstract}

Keywords: distributed compressive sensing, information fusion, face recognition, joint sparse representation

\section{Introduction}

Face recognition has a wide range of applications such as surveillance, information security, access control, identity fraud and so on. A typical face recognition system involves two main steps: feature extraction and classification. Subspace representation is the commonly used feature extraction method, such as principle component analysis (PCA) [1], linear discriminant analysis (LDA) [2] and so on. By using the extracted features, one can build classifiers based on various rules, such as nearest subspace method [3] and support vector machines [4] to perform face recognition. However, it is still a challenge problem to deal with face variations, such as expression, illumination and pose variation [5], for the above mentioned feature extraction methods.

Face recognition based on visual images have attracted many interests. However, the visible images are sensitive to illumination changes [6]. For the simple illumination and pose variations problem in visible images, some improved algorithms [7] have been proposed. However, these methods cannot overcome the complex environments without considering spectra images. Fortunately, some problems can be avoided using different types of sensors. For example, infrared sensors are less sensitive to ambient light as they capture the temperature of the body [8]. As a result, by using the infrared spectrum information of the infrared images, face recognition can potentially offer simpler but more robust solutions, improving the recognition performance in uncontrolled environments $[8,9]$.

In general, the infrared sensors are less sensitive to ambient light as they capture the temperature of the objects. However, the infrared images often lose information related to the texture of the face. In contrast to infrared images, the 
visible images are known more robust to the expression and pose variation but very sensitive to illumination changes. In this sense, we can simultaneously use different types of sensors to improve the robustness of the algorithms. Since the near infrared (NIR) and visible image can capture intrinsically different characteristics of the observed faces, intuitively, a better face description of images in a specific object could be constituted by utilizing the complementary information presented in the two spectra.

A more recent development for face recognition is based on the compressive sensing theory and has demonstrated good performance on robust face recognitions [10]. In this paper, under the sparsity assumption that an image can be expanded by a small number of training images of the same class, we propose a new method by fusing the information of the near infrared and visible images based on the recently emerged distributed compressive sensing (DCS) theory. The distributed compressive sensing is an improved compressive sensing theory for multi-signal ensembles with correlation structures that can recover the signals [11]. Particularly, in this paper, as the near infrared and visible images can be regarded as two different signals in DCS with the complementary characteristics provided by different sensors, DCS is applied to enhance the discriminating power for stable classifications by assembling the complementary structures of the near infrared and visible images. It is worth mentioning that, the condition for DCS method is the same as the compressive sensing methods. That is, if the training number of images is small, a least-square problem can be solved without the sparsity consideration. If the training number of images is large, the solution of an $\ell_{1}$ regularized problem is necessary. However, DCS outperforms compressive sensing in the sense that it considers more intrinsic structure information of the face images.

The rest of this paper is organized as follows. In Section 2, we briefly review the compressive sensing and distributed compressive sensing. In Section 3, we illustrate the details of the proposed method. The empirical studies on some benchmark datasets are presented in Section 4. Finally, we draw the conclusions in Section 5 .

\section{Compressive Sensing and Distributed Compressive Sensing}

Compressive sensing aims to solve the problem of acquiring an $n \times 1$ discrete time signal $\mathbf{f}$ that is $K$-sparse or compressible in some sparsity basis matrix $\Psi$ (where each column is a basis vector $\Psi_{j}$ ). $K$-sparse means that only $K \square n$ of the expansion coefficients $\mathbf{x}$ representing $\mathbf{f}=\mathbf{\Psi} \mathbf{x}$ are nonzero. By compressible, the representation $\mathbf{f}=\boldsymbol{\Psi x}$ has just a few large coefficients and many small coefficients. Candès et. al. demonstrate that the $K$-sparse or compressible signal can be recovered from $m=c K$ non-adaptive linear projections through the measurement matrix $\boldsymbol{\Phi}$, where $c$ is a small constant [12]. Furthermore, the condition of exact recovery is that the matrix $\boldsymbol{\Phi}$ is incoherent with $\boldsymbol{\Psi}$. The coherence between $\boldsymbol{\Phi}$ and $\boldsymbol{\Psi}$ can be computed by

$$
\mu(\boldsymbol{\Phi}, \boldsymbol{\Psi}):=\sqrt{N} \sqsubset \max _{\substack{1 \leq i \leq m \\ 1 \leq j \leq N}}\left|<\Phi_{i}, \Psi_{j}>\right|
$$


Rather than directly measuring the $n$-point signal f , compressive sensing only samples a much compressed signal $\mathbf{y}$ via a linear projection over the original signal f . More specifically, let $\mathbf{D}=\boldsymbol{\Phi} \Psi \in R^{k \times n}$ and $\mathbf{y}=\boldsymbol{\Phi f}=\boldsymbol{\Phi} \Psi \mathbf{x}=\mathbf{D x}$. Since $K \square n$, recovering the signal $\mathbf{f}$ from the measurements $\mathbf{y}$ is ill-posed problem. However, with the additional assumption of signal sparsity under the basis $\boldsymbol{\Psi}$, we can make the exact recovery under some conditions. In mathematics view, $\mathbf{x}$ can be recovered by solving the linear inverse problem through $\ell_{0}$ optimization problem

$\mathbf{x}=\min \|\mathbf{x}\|_{0}$ s.t. $\mathbf{y}=\mathbf{D} \mathbf{x}$

According to Candès' study [12], we only need $m=O(K \log (n / K))$ measurements, and then we can reconstruct the original signal $\mathbf{f}$ by $\mathbf{f}=\Psi \mathbf{x}$, where $\mathbf{x}$ is the sparse solution of the $\ell_{0}$-sparsest problem (2). And the problem (2) is commonly transformed to $\ell_{1}$ optimal problem and solved by linear programming. Compressive sensing (CS) enables a sparse or compressible signal to be reconstructed from a small number of non-adaptive linear projections, thus significantly reduces the sampling and computation costs [12]. CS has many promising applications in signal acquisition, compression and medical imaging.

Baron et. al. introduced a model for jointly sparse signals and proposed the corresponding joint reconstruction algorithms [13], known as the distributed compressive sensing (DCS) theory which is related to the joint sparsity of a signal ensemble. They demonstrated that the exact reconstruction of signals in DCS, while the sensors operate entirely without collaboration. It has dramatic savings relative to the measurement features required for separate CS decoding. As in the jointly sparse model [11], all signals could be decomposed into two parts. All signals share a common sparse component $\mathbf{z}_{C}$ while each individual signal contains a sparse innovation component $\mathbf{z}_{j}, j \in\{1,2, \ldots, J\}$, that is,

$$
\mathbf{y}_{j}=\mathbf{z}_{C}+\mathbf{z}_{j}, j \in\{1,2, \ldots, J\},
$$

where $\mathbf{z}_{C}=\boldsymbol{\Psi} \boldsymbol{\theta}_{C},\left\|\boldsymbol{\theta}_{C}\right\|_{0}=K_{C}$ and $\mathbf{z}_{j}=\boldsymbol{\Psi} \boldsymbol{\theta}_{j},\left\|\boldsymbol{\theta}_{j}\right\|_{0}=K_{j}$. Moreover, $\mathbf{z}_{C}$ is the common component to all of the $\mathbf{y}_{j}$ and has sparsity $K_{C}$ in basis $\boldsymbol{\Psi}$. The $\mathbf{z}_{j}$ are the unique portions of the $\mathbf{y}_{j}$ and have sparsity $K_{j}$ in the basis $\boldsymbol{\Psi}$. In addition to substantially reduced measurement rates in the process of applications, the DCSbased distributed source coding schemes share many of the attractive and intriguing properties of CS, particularly when we employ random projections at the sensors. As in CS, random measurement bases are universal in the sense that they can be paired with any sparse basis. Random coding is also robust. Thus they allow a progressively better reconstruction of the data as more measurements are obtained.

\section{The Proposed Method}

Using different types of sensors simultaneously could improve the robustness of the algorithms to most of complex circumstances. Since NIR and visible image can capture intrinsic characteristics of different component, in this paper, the 
complementary information in the two spectra can be utilized to enhance the stability. The framework of the proposed method is illustrated in Figure 1.

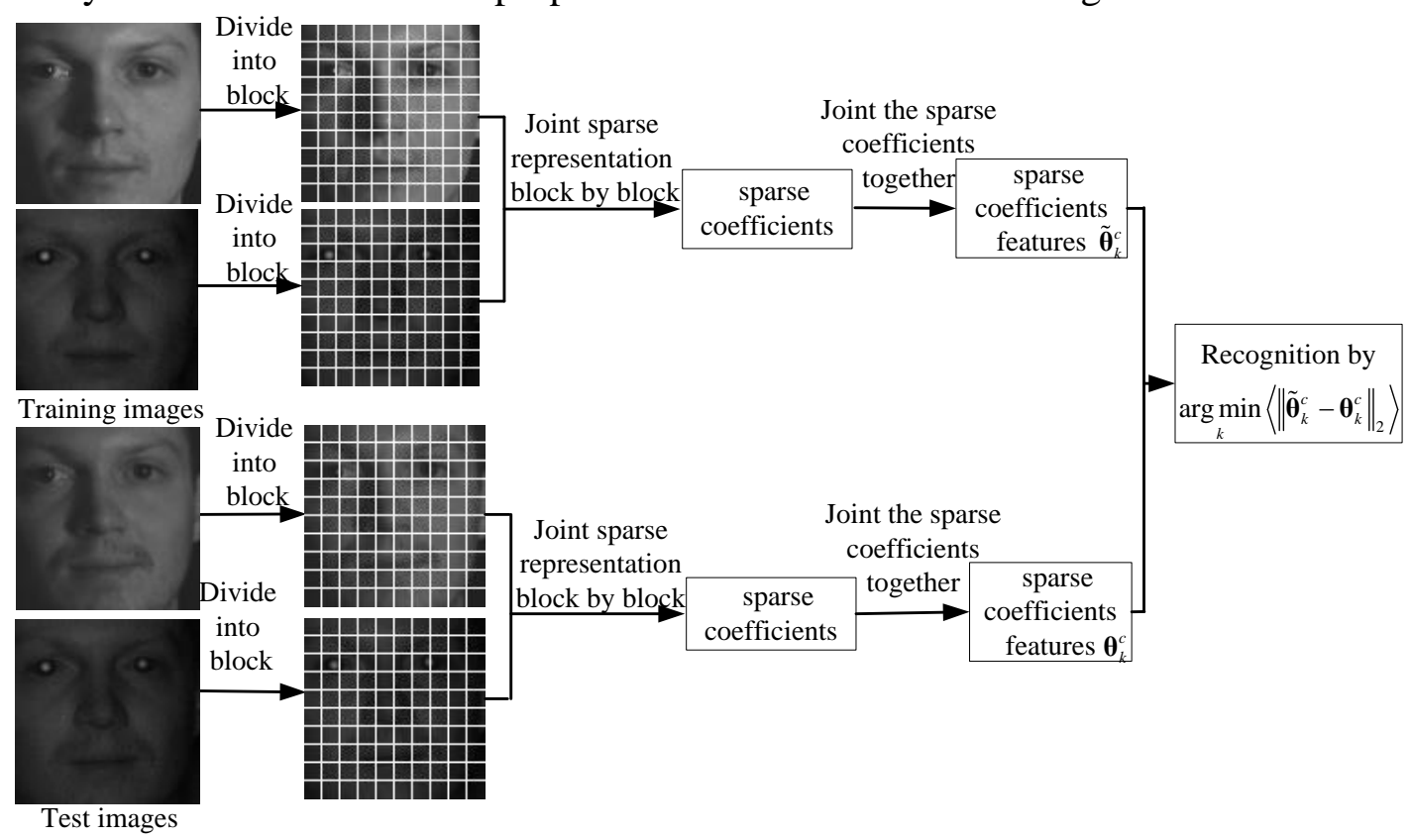

Figure 1. The Structure Chart of the Proposed Method

Specifically, we represent a NIR image and a visible image as 1-D column vector $\mathbf{y}^{n} \in R^{N}$ and $\mathbf{y}^{v} \in R^{N}$, respectively. We assume that there are $K$ distinct classes (i.e., subjects), with each class having $J_{k}$ training images, $k=1,2, \ldots, K$.

Let the images of class $k$ be represented as an ensemble $\left\{\mathbf{y}_{k, j}^{n}, \mathbf{y}_{k, j}^{v}\right\}, j=1, \ldots, J_{k}$. Hence, such an ensemble can be represented as $\mathbf{Y}=\left[\mathbf{y}_{k 1}^{n} \mathbf{y}_{k 2}^{v} \ldots \mathbf{y}_{k J_{k}}^{n} \mathbf{y}_{k J_{k}}^{v}\right]^{\mathrm{T}}$.

Notice that all signals in $\left\{\mathbf{y}_{k, j}^{n}, \mathbf{y}_{k, j}^{v}\right\}, j=1, \ldots, J_{k}$ for a given class $k$ are highly inter-correlated, we can represent the $j$-th training image in the class $k$ as the sum of a common component and an innovation component by $\mathbf{y}_{k j}^{n}=\mathbf{z}^{c}+\mathbf{z}_{k j}^{n i}$ and $\mathbf{y}_{k j}^{v}=\mathbf{z}^{c}+\mathbf{z}_{k j}^{v i}$, respectively. Furthermore, let $\boldsymbol{\Psi}$ be the matrix representation of some orthonormal basis (e.g., DCT, wavelet) that can sparsely represent the training images, so that coefficients of signal $\mathbf{y}$ can be written as,

$$
\mathbf{y}_{k j}^{n}=\mathbf{z}^{c}+\mathbf{z}_{k j}^{n i}=\boldsymbol{\Psi} \boldsymbol{\theta}_{k}^{c}+\boldsymbol{\Psi} \boldsymbol{\theta}_{k, j}^{n i}
$$

and

$$
\mathbf{y}_{k j}^{v}=\mathbf{z}^{c}+\mathbf{z}_{k j}^{v i}=\boldsymbol{\Psi} \boldsymbol{\theta}_{k}^{c}+\boldsymbol{\Psi} \boldsymbol{\theta}_{k, j}^{v i}
$$

Here $\boldsymbol{\theta}_{k}^{c}$ is the common component to all the $J_{k}$ training images of class $k$ and $\boldsymbol{\theta}_{k, j}^{n i}, \boldsymbol{\theta}_{k, j}^{v i}\left(j=1, \ldots, J_{k}\right)$ are the innovation component to NIR image and visible image respectively. Under this model, let the common and innovation components of class $k$ be jointly represented by the vector

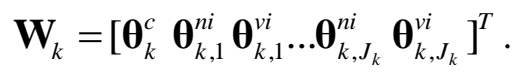


The sparse vector $\mathbf{W}_{k}$ can be found by solving the following $\ell_{0}$ minimization problem,

$\mathbf{W}=\min \left\|\mathbf{W}_{k}\right\|_{0}$ s.t. $\mathbf{Y}=\mathbf{D} \mathbf{W}_{k}$,

where $\mathbf{D}=\left[\begin{array}{ccccc}\boldsymbol{\Psi} & \boldsymbol{\Psi} & 0 & \cdots & 0 \\ \boldsymbol{\Psi} & 0 & \boldsymbol{\Psi} & \ldots & 0 \\ \vdots & \vdots & \vdots & \ddots & 0 \\ \boldsymbol{\Psi} & 0 & 0 & 0 & \boldsymbol{\Psi}\end{array}\right]$.

To solve problem (7), different algorithms can be used in practice, such as iterative soft thresholding algorithm (IST) [14] and gradient projection for sparse reconstruction (GPSR) algorithm [15]. In this paper, we select the GPSR method [15] to solve problem (7) due to its effectiveness and efficiency.

The coefficients $\boldsymbol{\theta}_{k}^{c}$, which gathered the common component of the near infrared and visible image, is obtained by (6). Then, the test object is classified based on the approximations by assigning it to the object class that minimizes the distance between $\tilde{\boldsymbol{\theta}}_{k}^{c}$ and $\boldsymbol{\theta}_{k}^{c}$ :

$q=\arg \min _{k}\left\langle\left\|\tilde{\boldsymbol{\theta}}_{k}^{c}-\boldsymbol{\theta}_{k}^{c}\right\|_{2}\right\rangle$.

In order to reduce the computing complexity of the algorithm, the images are divided into small blocks, and the algorithm is performed on these small blocks. In the end, we identify the class by the minimum sum of the distance. Algorithm 1 below summarizes the complete recognition procedure.

\section{Algorithm 1: Face recognition based on distributed compressive sensing}

Input: The near infrared image and visible image. Orthonormal basis $\boldsymbol{\Psi}$, (e.g., DCT).

1: Represent the near infrared image and visible image as 1-D column vector $\mathbf{y}_{k j}^{n} \in R^{N}, j=1, \ldots, J_{k}$ and $\mathbf{y}_{k j}^{v} \in R^{N}, j=1, \ldots, J_{k}$, respectively.

2: Represent the $j$-th training image of near infrared image and visible image as the sum of a common component and an innovation component respectively as follows, $\mathbf{y}_{k j}^{n}=\mathbf{z}^{c}+\mathbf{z}_{k j}^{n i}$ and $\mathbf{y}_{k j}^{v}=\mathbf{z}^{c}+\mathbf{z}_{k j}^{v i}$.

3: Let the common and innovation components of class $k$ be jointly represented by the vector $\mathbf{W}_{k}=\left[\begin{array}{llll}\boldsymbol{\theta}_{k}^{c} & \boldsymbol{\theta}_{k, 1}^{n i} & \boldsymbol{\theta}_{k, 1}^{v i} \ldots \boldsymbol{\theta}_{k, J_{k}}^{n i} & \boldsymbol{\theta}_{k, J_{k}}^{v i}\end{array}\right]^{T}$, which can be found by solving the $\ell_{0}$ minimization problem (7). And $\boldsymbol{\theta}_{k}^{c}$ is obtained by (6).

4: Do the same transformations on test images as in step 2, 3, 4, obtaining $\tilde{\boldsymbol{\theta}}_{k}^{c}$ for test images.

5: Classify the test object based on these approximations by assigning it to the object class that minimizes the distance between $\tilde{\boldsymbol{\theta}}_{k}^{c}$ and $\boldsymbol{\theta}_{k}^{c}$ by (8), and return class label $q$.

Output: Class label $q$. 
Furthermore, since we focus on the classification rather than the exact reconstruction of signals, we can extract the low dimension features to decrease the computation complexity. Let the dimensionality reduction matrix be $\boldsymbol{\Phi} \in R^{M \times N}$ ( $\boldsymbol{\Phi}$ can be random or any matrix highly incoherent with $\boldsymbol{\Psi}$ ), then, the low dimensional projection of the test image can be calculated as $\tilde{\mathbf{c}}=\boldsymbol{\Phi c} \in R^{M}$. Therefore, the low dimensional versions of the training features $\tilde{\mathbf{z}}_{k}^{c}$ and $\tilde{\mathbf{z}}_{k}^{i}$ are computed by $\tilde{\mathbf{z}}_{k}^{c}=\boldsymbol{\Phi} \mathbf{z}_{k}^{c}, \widetilde{\mathbf{z}}_{k}^{i}=\boldsymbol{\Phi} \mathbf{z}_{k}^{i}, \in R^{M}$. Then, the problem of (7) is computed using the modified problem as follows

$$
\mathbf{W}=\min \left\|\mathbf{W}_{k}\right\|_{0} \text { s.t. } \tilde{\mathbf{Y}}=\tilde{\mathbf{D}} \mathbf{W}_{k},
$$

where $\tilde{\mathbf{D}}=\left[\begin{array}{ccccc}\boldsymbol{\Phi} \Psi & \boldsymbol{\Phi} \boldsymbol{\Psi} & 0 & \ldots & 0 \\ \boldsymbol{\Phi} \boldsymbol{\Psi} & 0 & \boldsymbol{\Phi} \boldsymbol{\Psi} & \ldots & 0 \\ \vdots & \vdots & \vdots & \ddots & 0 \\ \boldsymbol{\Phi} \boldsymbol{\Psi} & 0 & 0 & 0 & \boldsymbol{\Phi} \Psi\end{array}\right]$

The recognition of the testing image can then be determined as algorithm 1 . The process of solving the sparse coefficient feature can be shown as distributed compressive sensing and it substantially reduces the measurement rates in multisignal application.

\section{Experiments}

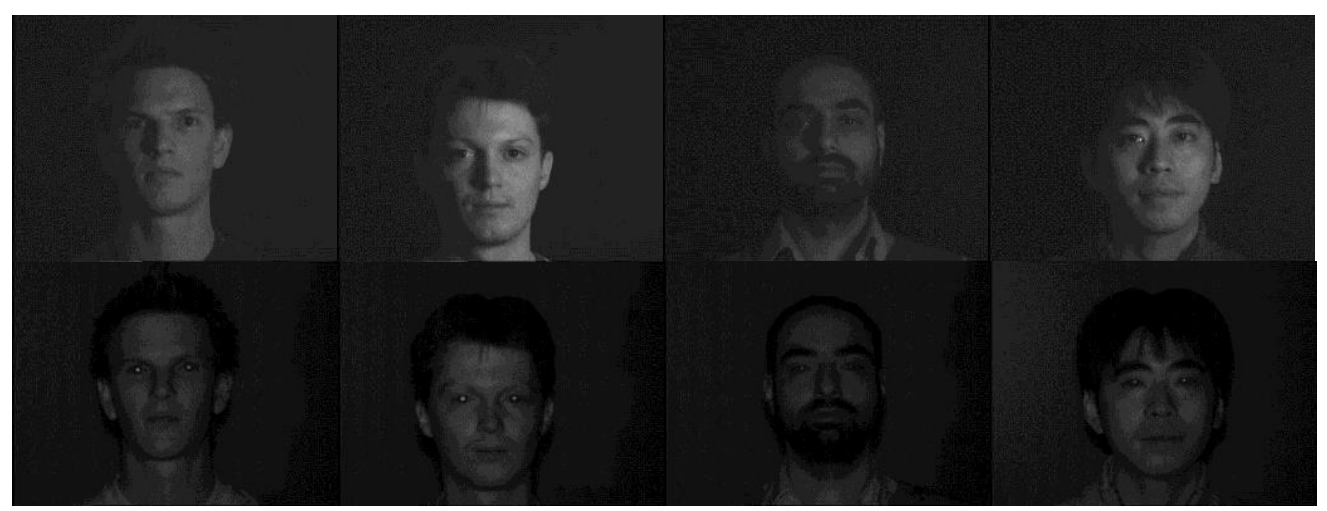

(a) The original images

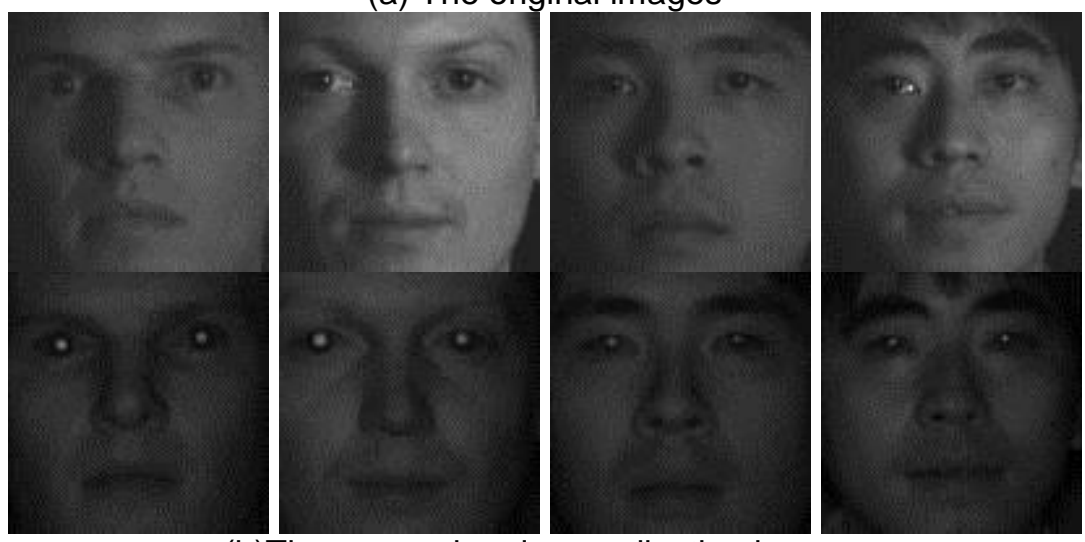

(b) The cropped and normalization images

Figure 2. Some Samples of Near Infrared and Visible Face Database from University of SURREY 
This section assesses the performance of the proposed method by using the near infrared and visible face database from the University of SURREY [16] and Harbin Institute of Technology Shenzhen Graduate School (HITSZ) [17]. In SURREY database, there are 1080 visible face images and 1080 NIR faces of 23 subjects. The NIR images are corresponding to the visible images for each object. The background in the SURREY face database was detected and cropped by SMQT algorithm [18]. The cropped face is then normalized to $100 \times 100$ pixels. Considering on testing the effectiveness of the algorithm in complex environments, we did not register images for this database. Figure 2 shows some samples of NIR and visible face database from university of SURREY. Figure 2 (a) is the original images, and (b) is the cropped and normalized face images. The above row is the visible images, and the near infrared images are at the bottom.

\section{A. Experiments on SURREY Database}

In order to reduce the computing complexity of the algorithm, the images are divided into $10 \times 10$ small block. We process the small blocks, and identify the class by the minimum sum of the distance. In the algorithm 1, the problem (7) is solved by GPSR algorithm and the parameter $\tau$ of GPSR is set as suggested in [14]. We set $\tau$ to $0.005\left\|\mathbf{D}^{T} \mathbf{y}\right\|_{\infty}$. In this paper, 4, 8, 12, 16, 20 images of each individual were selected randomly and used for training. The rest of the images were used for testing. All the experiments are repeated 10 times independently and the recognition rates are the mean of the 10 times.

In order to choose orthonormal basis $\boldsymbol{\Psi}$ for the algorithm 1, we compare the recognition rates with DCT basis and Haar wavelet basis in the first experiment. The recognition rates are shown in Table 1. From Table 1, we know that the recognition rates with DCT basis are better than Haar basis. So in the following experiments, DCT basis is used as the orthonormal basis $\boldsymbol{\Psi}$.

Table 1. The Recognition Rates with Different Orthonormal Basis (\%)

\begin{tabular}{|c|c|c|}
\hline Training Number & DCT & Haar \\
\hline 4 & 84.17 & 84.12 \\
\hline 8 & 91.13 & 89.51 \\
\hline 12 & 91.81 & 90.61 \\
\hline 16 & 94.08 & 93.26 \\
\hline 20 & 94.35 & 94.67 \\
\hline
\end{tabular}

Table 2. Recognition Rates of the Proposed Method Compare with Using Visible Images and NIR Image (\%)

\begin{tabular}{|c|c|c|c|}
\hline Training Number & Visible Image & $\begin{array}{c}\text { Near Infrared } \\
\text { Image }\end{array}$ & DCS \\
\hline 4 & 63.88 & 82.98 & $\mathbf{8 4 . 7 9}$ \\
\hline 8 & 77.81 & 89.49 & $\mathbf{9 1 . 0 3}$ \\
\hline 12 & 74.64 & 91.81 & $\mathbf{9 2 . 2 8}$ \\
\hline 16 & 78.20 & 93.02 & $\mathbf{9 3 . 1 5}$ \\
\hline 20 & 84.02 & 93.84 & $\mathbf{9 3 . 9 2}$ \\
\hline
\end{tabular}


In the second experiment, we perform the experiment to test the complementarities of NIR image and visible image. We compare our method with the face recognition with only visible image or near infrared image. In algorithm 1, the distance between sparse coefficients of the face images according to (8) is applied for face recognition. If we only use the visible or near infrared image, the distance of the face images is computed for face recognition. For this experiment, the average recognition rates of the considered methods on university of SURREY face database are shown in Table 2. In Table 2, we notice that the recognition rate of the proposed DCS method is higher than using only visible images or near infrared images. It demonstrates that visible and near infrared images have some complements. There is an enormous enhancement of the DCS method compared to the method with only visible image. And the recognition rates of the proposed DCS method are better than the method with only NIR images slightly.

Table 3. Recognition Rates with 10 Objects on University of SURREY Face Database (\%)

\begin{tabular}{|c|c|c|c|c|c|c|c|}
\hline $\begin{array}{c}\text { Training } \\
\text { Number }\end{array}$ & LBP & PCA & 2DPCA & SRC & LDA & $\begin{array}{c}\text { Gabor- } \\
\text { LDA }\end{array}$ & DCS \\
\hline 4 & 87.16 & 82.25 & 91.20 & 83.00 & 90.87 & $\mathbf{9 2 . 2 7}$ & 92.20 \\
\hline 8 & 93.75 & 90.55 & 93.56 & 88.75 & 93.15 & 94.97 & $\mathbf{9 5 . 0 0}$ \\
\hline 12 & 96.27 & 90.75 & 95.41 & 95.00 & 94.45 & 96.07 & $\mathbf{9 6 . 5 8}$ \\
\hline 16 & 96.25 & 91.00 & 95.63 & 95.00 & 94.56 & 96.21 & $\mathbf{9 8 . 2 5}$ \\
\hline 20 & 97.57 & 92.75 & 96.25 & 97.50 & 95.25 & 98.16 & $\mathbf{9 8 . 2 5}$ \\
\hline
\end{tabular}

Table 4. Recognition Rates with 23 Objects on University of SURREY Face Database (\%)

\begin{tabular}{|c|c|c|c|c|c|c|c|}
\hline $\begin{array}{c}\text { Training } \\
\text { Number }\end{array}$ & LBP & PCA & 2DPCA & SRC & LDA & $\begin{array}{c}\text { Gabor- } \\
\text { LDA }\end{array}$ & DCS \\
\hline 4 & 84.57 & 54.87 & 79.13 & 71.96 & 79.21 & $\mathbf{8 5 . 6 1}$ & 84.79 \\
\hline 8 & 91.03 & 63.72 & 87.01 & 75.49 & 81.60 & 88.76 & $\mathbf{9 1 . 0 3}$ \\
\hline 12 & 89.13 & 68.12 & 91.19 & 77.75 & 85.75 & 91.96 & $\mathbf{9 2 . 2 8}$ \\
\hline 16 & 92.39 & 70.87 & 92.34 & 83.91 & 87.64 & 92.74 & $\mathbf{9 3 . 1 5}$ \\
\hline 20 & 91.31 & 76.41 & 92.61 & 88.26 & 90.45 & 93.54 & $\mathbf{9 3 . 9 2}$ \\
\hline
\end{tabular}

In the third experiment, the results of the proposed DCS method are compared with local binary pattern (LBP) method [19], PCA method [1], 2DPCA method [20], sparse representation based face classification algorithm (SRC) [10], linear discriminant analysis (LDA) [21] and Gabor-LDA method [22]. Form Table 2, we can observe that the recognition rate of NIR images can be significantly higher than the visible images. Therefore, in the following experiments, we use the NIR images for the single component experiments. The $\chi^{2}$ distances between LBP histograms are used for LBP feature based face recognition. In PCA method, we choose 30 discriminant features for the representation of each face image and use Euclidean distance to compare the face. In order to improve the recognition rate of SRC, the images were first segmented into blocks of size $10 \times 10$ pixel, and the blocks are used for the recognition. We present the recognition performance with variation in number of training images and training objects. Table 3 and Table 4 list the average recognition rates of the considered methods on SURREY database 
with 10 and 23 training objects, respectively. We list the results with respect to different numbers of training images for each table. From Table 3 and Table 4, we can see that the recognition rate increases with the increasing of training images number. What's more, for different size of the training images, the recognition rate is different. LBP and SRC method show good performance when the training number is relatively small. However, their performance becomes worse than DCS method when the training number of images becomes large. Although the LDA and Gabor-LDA method perform well when training number of images is 4 , their performance becomes worse than DCS method when the training number is increasing.

To demonstrate the performance of our algorithm in low dimensional feature space, we apply linear random measurement on training images and test images to retain only $50 \%$ of the original features (feature space of 50 points). We compare the recognition rates with 10 objects and 23 objects, respectively. The average recognition rates are shown in Figure 3 and Figure 4. From these figures, we can observe that the performance of the proposed DCS method can be also very stable when the low dimension features are used.

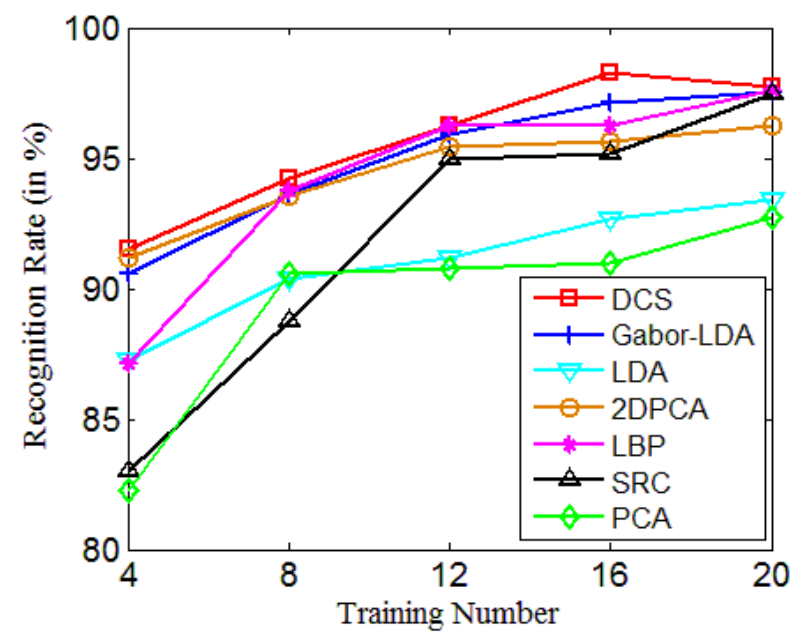

Figure 3. Recognition Rates in Low Dimensional Feature Space for 10 Objects

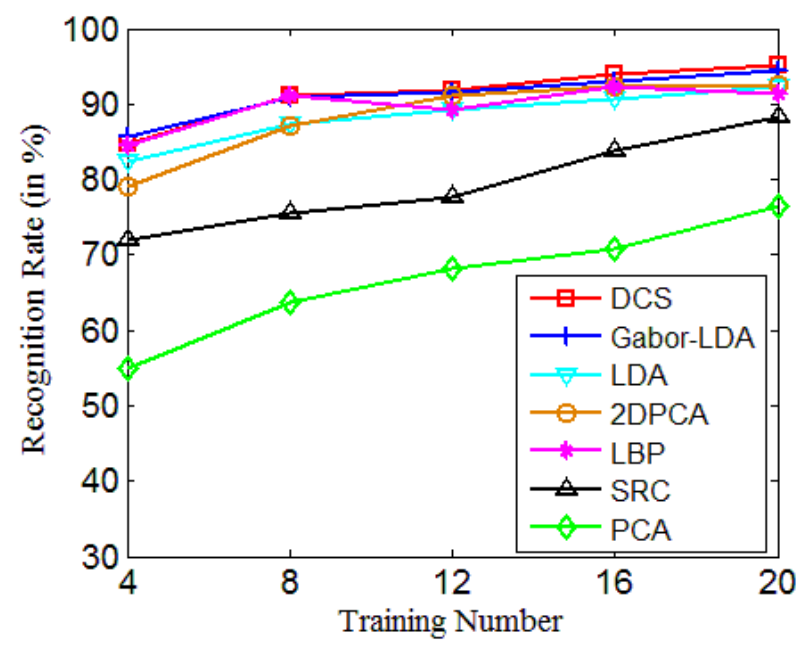

Figure 4. Recognition Rates in Low Dimensional Feature Space for 23 Objects 


\section{B. Experiments on HITSZ Database}

HITSZ near infrared and visible face database includes two datasets. In our paper, we use Lab2, which contains visible and near-infrared images of the 50 volunteers (subjects). There are 20 visible face images and the same number of near-infrared face images for each subject. These images were acquired under different illumination conditions. Figure 5 shows some NIR and visible samples from HITSZ. The top row shows the near infrared images, and the bottom shows the visible images. We perform two experiments on this database. The parameters are set to the same as the experiments on SURREY database. We perform two experiments on this database. The first experiment is to test the performance of the DCS method on original features. The second one is to demonstrate the performance of the DCS method in low dimensional feature space. Both the experiments are repeated 10 times independently and the recognition rates are the mean of the 10 times.

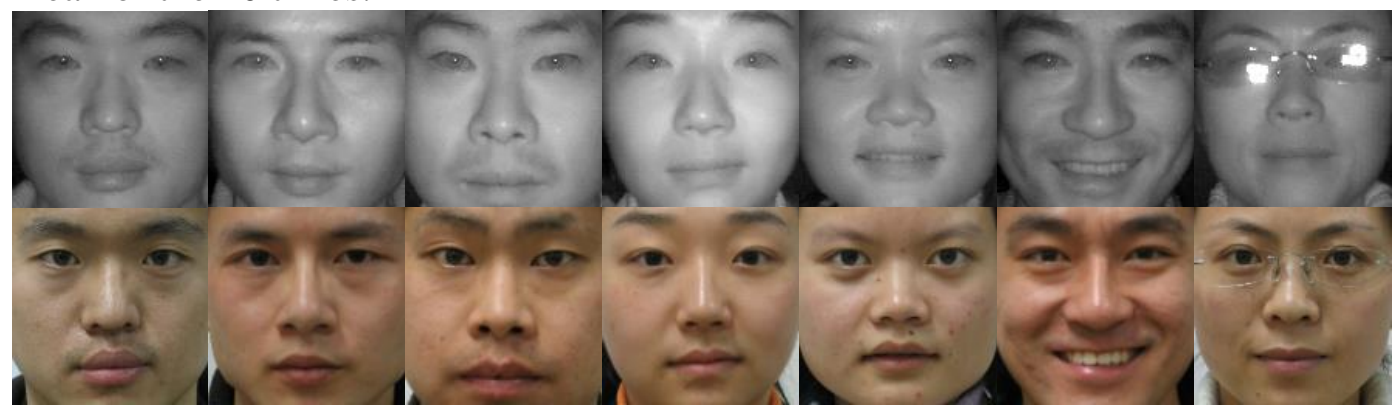

Figure 5. Some Samples of Near Infrared and Visible Face Database from HITSZ Database

Table 5. Recognition Rates on HITSZ Face Database (\%)

\begin{tabular}{|c|c|c|c|c|c|c|c|}
\hline $\begin{array}{c}\text { Training } \\
\text { Number }\end{array}$ & LBP & PCA & 2DPCA & SRC & LDA & $\begin{array}{c}\text { Gabor- } \\
\text { LDA }\end{array}$ & DCS \\
\hline 5 & 83.24 & 74.34 & 79.37 & 77.36 & 78.32 & $\mathbf{8 5 . 3 4}$ & 85.31 \\
\hline 10 & 84.19 & 78.17 & 84.53 & 80.84 & 83.69 & 87.64 & $\mathbf{8 8 . 4 9}$ \\
\hline 15 & 86.75 & 81.68 & 86.94 & 87.83 & 84.53 & 88.37 & $\mathbf{8 9 . 9 2}$ \\
\hline
\end{tabular}

For each subject, we randomly select 5, 10, 15 images from one subject for training, and the rest for testing, respectively. For comparison, we implemented the following seven methods: LBP [19], PCA, 2DPCA [20], SRC [10], LDA [21] and Gabor-LDA [22]. Table 5 presents the experimental results. From Table 5, although, the recognition accuracy of Gabor-LDA method is higher than others when the training number of images is 5, the recognition accuracy of the proposed DCS method can be significantly higher than other methods when the training number is 10 or 15 .

Furthermore, to demonstrate the performance of our algorithm in low dimensional feature space, we applies linear random measurement on training images and test images to retain only $50 \%$ of the original features (feature space of 50 points). Experimental results are shown in Table 6 . We can observe that, the recognition accuracy of Gabor-LDA method is higher than others when the training number is 5 , while the recognition rate of the proposed DCS method is much higher than other methods when the training number is 10 or 15 . On two 
databases, SURREY dataset and HITSZ dataset, although the LDA and GaborLDA method can obtain better performance when the training number is relatively small, when the training number is increasing, the recognition rate of the DCS method can be much higher than them.

\section{Table 6. Recognition Rates of HITSZ Face Database in Low Dimensional Feature Space (\%)}

\begin{tabular}{|c|c|c|c|c|c|c|c|}
\hline $\begin{array}{c}\text { Training } \\
\text { Number }\end{array}$ & LBP & PCA & 2DPCA & SRC & LDA & $\begin{array}{c}\text { Gabor- } \\
\text { LDA }\end{array}$ & DCS \\
\hline 5 & 81.62 & 73.75 & 78.73 & 76.43 & 77.82 & $\mathbf{8 4 . 7 4}$ & 84.39 \\
\hline 10 & 82.75 & 76.92 & 83.95 & 79.47 & 82.56 & 86.59 & $\mathbf{8 7 . 7 3}$ \\
\hline 15 & 84.43 & 80.94 & 85.75 & 87.93 & 83.47 & 87.63 & $\mathbf{8 8 . 4 7}$ \\
\hline
\end{tabular}

\section{Conclusions}

In this paper, we propose a novel face recognition method based on the distributed compressive sensing. In this method, visible and NIR images are fused by distributed compressive sensing for face recognition. The sparse coefficients extracted according to the distributed compressive sensing method can well assemble the common component of visible and NIR image, which can better utilize the information of the two component images. Experiments on SURREY and HITSZ face database show that the proposed method outperforms state-of-theart methods in terms of recognition rate.

\section{Acknowledgments}

Supported by Shanghai Training Funds for Young Teacher (No. ZZGCD15040).

\section{References}

[1] M. Turk and A. Pentland, "Eigenfaces for recognition", J. Cog. Neurosci., vol. 3, no. 1, (1991), pp. 7186.

[2] P. N. Belhumeur, J. P. Hespanha and D. J. Kriegman, "Eigenfaces vs. Fisherfaces: Recognition using class specific linear projection”, IEEE T. Pattern Anal., vol. 19, no. 7, (1997), pp. 711-721.

[3] J. Ho, M. Yang, J. Lim, K. Lee and D. Kriegman, "Clustering appearances of objects under varying illumination conditions", Proceedings of the CVPR, Madison, Wisconsin, (2003) June 16-22.

[4] J. A. Gualtieri and R. F. Cromp, "Support vector machines for hyperspectral remote sensing classification", Proceedings of the SPIE, (1998), San Diego, CA.

[5] W. Zhao, R. Chellappa, P. J. Phillips and A. Rosenfeld, "Face recognition: a literature survey", ACM Comput. Surv., vol. 35, no. 4, (2003), pp. 399-458 .

[6] Y. Adini, Y. Moses and S. Ullman, "Face recognition: the problem of compensating for changes in illumination direction", IEEE T. Pattern Anal., vol. 19, no. 7, (1997), pp. 721-731.

[7] D. H. Wang, X. K. Wang and S. Kong, "Integration of multi-feature fusion and dictionary learning for face recognition", Image Vision Comput., vol. 31, no. 12, (2013), pp. 895-904.

[8] W. Hizem, L. Allano, A. Mellakh and B. Dorizzi, "Face recognition from synchronised visible and nearinfrared images", IET Signal Process., vol. 3, no. 4, (2009), 282-289.

[9] L. Zhang, W. Zhang, L. Sun and Z. Fang, "Infrared Face Recognition Based on Compressive sensing and PCA", Proceedings of IEEE Computer Science and Automation Engineering, Shanghai, China, (2011) June 10-12.

[10] J. Wright, A. Y. Yang, A. Ganesh, S. S. Sastry and Y. Ma, "Robust face recognition via sparse representation", IEEE T. Pattern Anal., vol. 31, no. 2, (2009), pp. 210-227.

[11] D. Baron, M. B. Wakin, M. Duarte, S. Sarvotham and R. Baraniuk, "Distributed compressed sensing", Technique report, TREE-0612, Rice University, Department of Electrical and Computer Engineering, (2006). 
[12] E. Candés, J. Romberg and T. Tao, "Robust uncertainty principles: Exact signal reconstruction from highly incomplete frequency information”, IEEE T. Inform. Theory, vol. 52, no. 2, (2006), pp. 489-509.

[13] D. Baron, M. F. Duarte, S. Sarvotham, M. B. Wakin and R. G. Baraniuk, "An information-theoretic approach to distributed compressed sensing", Proceedings of the 43rd Allerton Conference Communication, Control, and Computing, Monticello, IL, (2005) September 28 - 30.

[14] I. Daubechies, M. Defrise and C. D. Mol, "An iterative thresholding algorithm for linear inverse problems with a sparsity constraint", Communications on Pure and Applied Mathematics, vol. 57, no. 11, (2004), pp. 1413-1457.

[15] M. A. T. Figueiredo, R. D. Nowak and S. J. Wright, "Gradient projection for sparse reconstruction: Application to compressed sensing and other inverse problems", IEEE J. Sel. Top. Signal Process., vol. 1 , no. 4, (2007), pp. 586-597.

[16] X. Zou, "Illumination Invariant Face Recognition Based on Active Near-Infrared Differential Imaging", University of Surrey, (2007)

[17] Y. Xu, A. Zhong, J. Yang and D. Zhang, "Bimodal biometrics based on a representation and recognition approach", Opt. Eng., vol. 50, no. 3, (2011).

[18] M. Nilsson, J. Nordberg and I. Claesson, "Face detection using local SMQT features and split up snow classifier", Proceedings of the ICASSP, Hawaii, USA, (2007) April 15-20.

[19] S. Z. Li, L. Zhang, S. C. Liao, X. X. Zhu and R. F. Chu, "A near-infrared image based face recognition system", Proceedings of the 7th International Conference on Automatic Face and Gesture Recognition, Southampton, UK, (2006) April 10-12.

[20] J. Yang, D. Zhang, A. F. Frangi and J. Y. Yang, "Two-dimensional PCA: a new approach to appearance based face representation and recognition", IEEE T. Pattern Anal., vol. 26, no. 1, (2004), pp. 131-137.

[21] J. Lu, K. N. Plataniotis, A. N. Venetsanopoulos, "Face Recognition Using LDA-Based Algorithms", IEEE T. Neural Networ., vol. 14, no. 1, (2003), pp. 195-200.

[22] Y. Pang, L. Zhang, M. Li, Z. Liu and W. Ma, "A Novel Gabor-LDA Based Face Recognition Method", Proceedings of Pacific-Rim Conference on MultiMedia, Tokyo, Japan, (2004) November 30-December 3.

\section{Author}

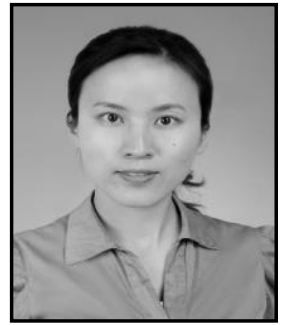

Dan Wei received the B.S., M.S., and Ph.D. degrees from Hunan University, Changsha, China, in 2006, 2008, and 2012, respectively. Her current research interests include image processing and pattern recognition. Now she is a lecturer in the College of Automotive Engineering, Shanghai University of Engineering Science. 